\title{
Genotype-by-sex interaction in the aetiology of type 2 diabetes mellitus: support for sex-specific quantitative trait loci in Hypertension Genetic Epidemiology Network participants
}

\author{
C. L. Avery • B. I. Freedman • A. T. Kraja • \\ I. B. Borecki • M. B. Miller • J. S. Pankow • D. Arnett • \\ C. E. Lewis • R. H. Myers • S. C. Hunt • K. E. North \\ Received: 15 December 2005 / Accepted: 18 June 2006 / Published online: 12 August 2006 \\ (C) Springer-Verlag 2006
}

\begin{abstract}
Aims/hypothesis While there are sex-related differences in both the prevalence of type 2 diabetes mellitus and disease risk factors, there is only limited research on sexspecific influences on type 2 diabetes aetiology within the same study population. Thus, we assessed genotypeby-sex interaction using a liability threshold model in an attempt to localise sex-specific type 2 diabetes quantitative trait loci (QTLs).

Subjects, materials and methods Hypertensive siblings and their offspring and/or parents in the Hypertension Genetic Epidemiology Network of the Family Blood Pressure Program were recruited from five field centres. The diabetic phenotype was adjusted for race, study centre, age and non-
\end{abstract}

Electronic supplementary material Supplementary material is available to this article at http://dx.doi.org/10.1007/s00125-006-0375-4 and is accessible to authorized users.

\footnotetext{
C. L. Avery $\cdot$ K. E. North $(\bowtie)$

Department of Epidemiology, CB \#8050,

The University of North Carolina,

Chapel Hill, NC 27514, USA

e-mail: kari_north@unc.edu

\section{B. I. Freedman}

Department of Internal Medicine, Wake Forest University,

Winston Salem, NC, USA
}

\section{A. T. Kraja $\cdot$ I. B. Borecki \\ Division of Statistical Genomics, \\ Washington University School of Medicine, \\ St Louis, MO, USA}

\section{B. Miller · J. S. Pankow}

Division of Epidemiology and Community Health,

University of Minnesota,

Minneapolis, MN, USA linear age effects. In total, 567 diabetic individuals were identified in 385 families. Variance component linkage analyses in the combined sample and stratified by sex and race were performed (SOLAR program) using race-specific marker allele frequencies derived from a random sample of participants at each centre.

Results We observed a QTL-specific genotype-by-sex interaction $(p=0.009)$ on chromosome 17 at $31 \mathrm{cM}$, with females displaying a robust adjusted logarithm of odds (LOD) of 3.0 compared with 0.2 in males and 1.3 in the combined sample. Three additional regions demonstrating suggestive evidence for linkage were detected: chromosomes 2 and 5 in the female sample and chromosome 22 (adjusted $\mathrm{LOD}=1.9$ ) in the combined sample. 
Conclusions/interpretation These findings suggest that multiple genes may regulate susceptibility to type 2 diabetes, demonstrating the importance of considering the interaction of genes and environment in the aetiology of common complex traits.

Keywords Genome scan - Genotype-by-sex interaction . Type 2 diabetes mellitus

\begin{tabular}{|c|c|}
\hline \multicolumn{2}{|c|}{ Abbreviations } \\
\hline ARP & affected relative pair \\
\hline $\mathrm{cM}$ & centimorgan \\
\hline ESM & Electronic Supplementary Material \\
\hline GRR & Graphical Representation of Relationships \\
\hline HyperGEN & $\begin{array}{l}\text { Hypertension Genetic Epidemiology Net- } \\
\text { work (of the Family Blood Pressure Program) }\end{array}$ \\
\hline IBD & identical-by-descent \\
\hline LOD & logarithm of odds \\
\hline$M A P 2 K 4$ & mitogen-activated protein kinase 4 gene \\
\hline NCORI & nuclear receptor corepressor 1 gene \\
\hline PPARG & peroxisome proliferator-activated receptor $\gamma$ \\
\hline QTL & quantitative trait locus \\
\hline
\end{tabular}

\section{Introduction}

Susceptibility to type 2 diabetes mellitus is determined by multiple genetic and environmental factors and interactions between the two. While there are sex-related differences in both the prevalence of type 2 diabetes and disease risk factors, research on complex interactions by sex in the pathogenesis of type 2 diabetes is limited [1,2]. Whether sex-specific genetic effects contribute to differences in type 2 diabetes status also warrants further investigation [3-5].

Modelling the true complexity of type 2 diabetes may advance our understanding of the aetiology of this major disease. For example, the identification of genotype-by-sex interactions could improve the localisation of type 2 diabetes quantitative trait loci (QTLs) and increase the support for linkage. Such findings may also help explain why females have a higher prevalence of type 2 diabetes in some populations [6-8], but not others [9].

We previously detected QTLs for type 2 diabetes status on chromosomes 22, 8 and 17 [10]. Since that previous study did not consider complex patterns of interaction by sex, and given that such patterning has been suggested in the literature reviewed above, we examined the evidence for genotype-by-sex interaction in the liability to type 2 diabetes by reanalysing a sample of siblings and/or their first-degree relatives from the HyperGEN (Hypertension Genetic Epidemiology Network of the Family Blood Pressure Program) cohort. The HyperGEN population may be ideal for further investigation of type 2 diabetes candidate genes considering that genetic heterogeneity (e.g. alleles at multiple loci conferring susceptibility to the trait of interest) complicates the identification of genes influencing common chronic diseases like type 2 diabetes by reducing study power. Thus, the heterogeneous nature of the disease indicates that homogeneous subgroups such as HyperGEN may represent the best strategy for susceptibility gene identification [11].

\section{Subjects, materials and methods}

Participants

This study examined participants in HyperGEN. Informed consent was obtained from all participants and this project was approved by the Institutional Review Boards of all participating institutions. HyperGEN methods [12] and eligibility criteria for this analysis [10] have been reported previously. Briefly, hypertensive siblings and their offspring and/or parents were recruited from five field centres located in the States of Massachusetts, North Carolina, Minnesota, Utah and Alabama. Sibship ascertainment criteria required sibships of two or more siblings with hypertension (defined as blood pressure $\geq 140 / 90 \mathrm{mmHg}$ or self-reported use of antihypertensive medications) in which age at hypertension diagnosis did not exceed 60 years.

Phenotyping

Participants were classified as having a diabetic phenotype if they reported using hypoglycaemic medications and/or their fasting serum glucose concentrations exceeded $7 \mathrm{mmol} / \mathrm{l}$. Individuals reporting an age at diagnosis $<30$ years were excluded from analyses in an attempt to remove type 1 diabetic persons. Serum glucose concentrations were measured with the Elan Glucose Reagent [13]. Briefly, the hexokinase phosphorylates glucose with ATP, producing glucose-6-phosphate. The product is then oxidised by glucose-6-phosphate dehydrogenase to form 6-phosphogluconate, with concurrent reduction of NAD to NADH. The ensuing increase in absorbance at $340 \mathrm{~nm}$ is directly related to the concentration of glucose in the sample.

\section{Genotyping}

Genotyping was performed by the NHLBI Mammalian Genotyping Service (Marshfield, WI, USA) (http://research. marshfieldclinic.org/genetics/, last accessed March, 2006). Cooperative Human Linkage Centre screening set eight was used, which included 387 microsatellite markers spaced 
(approximately 10 centimorgan [cM] distance) throughout the genome. Average marker heterozygosity was $77.7 \%$.

Quality control in several aspects on markers was performed. Relationship status among siblings was evaluated applying ASPEX, a likelihood-based method [14]. Mapmaker/Sibs [15] and PedCheck [16] were utilised to find and eliminate any Mendelian marker inconsistencies. Additionally, we have now completed advanced pedigree cleaning using the Graphical Representation of Relationships (GRR) program [17], where relationship mis-specifications are detected by pair-wise identity-by-state allele sharing. Using GRR, we determined if a reported pair-wise relationship was consistent with genotype data, and if not, we identified the most likely relationship given the data. We used the University of California Santa Cruz (http:// genome.ucsc.edu, last accessed March, 2006) and the Wellcome Trust Sanger Institute (http://www.sanger.ac.uk/, last accessed March, 2006) websites to determine the cytogenic location of markers and to search for candidate genes.

A loss of genotype data summary in the phase I HyperGEN sample is as follows: genotyping failure (4.3\%), family structure corrections and removal of monozygotic twin marker data $(1.16 \%)$, genotyping error and removal of marker data due to inconsistent allele calling within families $(0.71 \%)$, and inconsistent allele calling (1.42\%). Thus, the total loss of genotype data due to genotyping problems, genotyping errors and the cleaning process was $7.59 \%$

\section{Statistical genetic methods}

Linkage of type 2 diabetes status was examined using a variance component model in the combined sample and in each sex separately. Genome-wide logarithm of odds (LOD) scores were estimated using the program package SOLAR [18]. This approach is applicable to dichotomous traits under the assumption that an individual is classified as affected if an underlying genetically influenced liability, which is presumed to have a multivariate normal distribution, exceeds a given threshold [19].

Linkage to type 2 diabetes status was also tested using the variance component linkage model extended to include genotype-by-sex interaction at a QTL [20-22]. The expected genetic covariance between male and female relative pairs $(i, j)$ is: $\operatorname{Cov}\left(g_{\mathrm{iM}}, g_{\mathrm{jF}}\right)=2 \phi_{i j} \rho_{g(\mathrm{M}, \mathrm{F})} \sigma_{g \mathrm{M}} \sigma_{g \mathrm{~F}}+$ $\pi_{i j} \sigma_{q \mathrm{M}} \sigma_{q \mathrm{~F}}$, where the subscripts $\mathrm{M}$ and $\mathrm{F}$ refer to male and female, $\phi$ is the coefficient of kinship between two individuals, $\left.\rho_{g(\mathrm{M},}, \mathrm{F}\right)$ is the additive genetic correlation between the expressions of the trait in the two sexes, $\sigma_{\mathrm{gM}}$ and $\sigma_{g \mathrm{~F}}$ are the genetic SDs for males and females, $\pi_{i j}$ is the probability that individuals $i$ and $j$ are identical-by-descent (IBD) at a QTL linked to a genetic marker locus, and $\sigma_{q \mathrm{M}}$ and $\sigma_{q \mathrm{~F}}$ are the marker-specific genetic SDs for males and females, respectively.
The absence of additive genotype-by-sex interaction (the null hypothesis) corresponds to the genetic correlation between a male and female relative pair equalling one $\left(\rho_{g(\mathrm{M}, \mathrm{F})}=1.0\right)$. Male and female genetic SDs should also be equivalent under the null hypothesis $\left(\sigma_{g \mathrm{M}}=\sigma_{g \mathrm{~F}}\right)$. Each hypothesis is evaluated separately by comparing the likelihood of a model with one parameter constrained to the likelihood of the full model with all parameters estimated. The test statistic in analyses examining genetic correlation is a mixture of chi-square with one degree of freedom and a point mass at zero, as the parameter is constrained to a boundary. In the case of the additive genetic variances, the test statistic is a one degree of freedom chi-square, as no boundary is involved. Although there is a compound null hypothesis, $\rho_{g}=1$ and $\sigma_{g \mathrm{M}}=\sigma_{g \mathrm{~F}}$, we tested for different magnitudes of additive genetic variance and different sources of additive genetic variance individually in separate likelihood ratio tests that each have a single parameter constrained. For example, when testing for $\rho_{g}<1$, separate additive genetic variances for males and females are estimated and when testing for different additive genetic variances $\rho_{g}$ is allowed to float. The null hypothesis of no genotype-bysex interaction is rejected if either of these tests is significant at $\alpha=0.05$.

In addition, we added the marker-specific parameters to the model $\left(\sigma_{q \mathrm{M}}, \sigma_{q \mathrm{~F}}\right)$ and tested for QTL-specific genotypeby-sex interaction. Marker-specific genotype-by-sex interaction is indicated when the model likelihood in which the male and female marker-specific SDs are constrained to be equal $\left(\sigma_{q \mathrm{M}}=\sigma_{q \mathrm{~F}}\right)$ (i.e. the null hypothesis) is compared with the likelihood of a general model, in which all additive and QTL-specific parameters are estimated. All genotype-bysex interactions were assessed using likelihood-ratio tests ( $\alpha=0.05$ ) that compare the likelihood of a model that includes a genotype-by-sex interaction parameter against a model that restricts genotype-by-sex interaction [23-25].

Although linkage analysis is robust to ascertainment, accounting for sampling and ascertainment can increase the power to detect a QTL [26] and improves generalisability of linkage findings to the general population [27]. Because the HyperGEN recruitment protocol required sibships of two or more hypertensive persons, an exact ascertainment correction for proband status was unavailable. Therefore, we chose one randomly selected proband from each family, as Comuzzie and Williams [26] demonstrated that this correction is easily implemented and appears highly effective in improving the estimation of population parameters and decreasing type II error.

Linkage findings were evaluated by examining the empirical distribution of the LOD scores, under the assumption of multivariate normality, using 10,000 replicates and simulation methods incorporated into SOLAR [18]. The empirical distribution of the simulated LOD 
scores was used to assign percentiles to each replicate and estimate an expected test statistic. SOLAR then produced a constant to correct for the non-normality of the phenotype by regressing the expected LOD scores on the simulated LOD scores, which were then used to determine the adjusted genome-wide LOD scores (adjusted LOD score $=$ observed LOD score $\times$ correction constant) [28].

The variance component approach required IBD matrix estimates. The small family sizes of HyperGEN participants permitted us to calculate exact conditional probabilities employing the Lander-Green algorithm as implemented in MERLIN [29]. Allele frequencies from a random sample of participants at each field centre were calculated separately in the African-American and Caucasian groups. The IBD probabilities computed by MERLIN were then combined into a single set of multipoint IBD files in the SOLAR format using the program Mer2sol (http://taxa.epi.umn.edu/ mer2sol/, last accessed March, 2006) developed by Michael B. Miller at the University of Minnesota.

\section{Application to HyperGEN data}

The diabetic phenotype was adjusted for race, centre, age and non-linear age effects (see Table 1 of the Electronic Supplementary Material [ESM]). While we also examined the influence of adiposity by adjusting for percentage body fat, the main conclusions were unaltered. No additional covariate adjustments were made, since we investigated prevalent type 2 diabetes status and did not know the true covariate values at disease onset.

\section{Results}

The characteristics of covariates in the combined sample and stratified by sex are shown in Table 1 and the distribution of relative pairs in the combined sample and
Table 2 Distribution of relative pairs among 3,153 HyperGEN participants in the combined sample and stratified by sex for all participants and affected individuals only

\begin{tabular}{llll}
\hline Relative pair & Combined sample & Females & Males \\
\hline All participants $(n=3,153)$ & & & \\
Total & 3,947 & 1,380 & 813 \\
Parent-offspring & 652 & 251 & 112 \\
Siblings & 1,931 & 682 & 417 \\
Avuncular & 823 & 252 & 172 \\
Half-siblings & 218 & 90 & 40 \\
First cousins & 194 & 45 & 58 \\
Other & 129 & 60 & 14 \\
Affected individuals only & & & \\
(n=567) & & & \\
Total & 155 & 69 & 30 \\
Parent-offspring & 27 & 16 & 4 \\
Siblings & 112 & 46 & 22 \\
Avuncular & 4 & 2 & 1 \\
Half-siblings & 12 & 5 & 3 \\
First cousins & 0 & 0 & 0 \\
Other & 0 & 0 & 0 \\
\hline
\end{tabular}

by sex is presented in Table 2. Eighty percent of the 567 $(n=412)$ participants classified as diabetic reported hypoglycaemic medication use. Sibling relative pairs were most often represented due to the study design. Five hundred African-American and 419 Caucasian families were available for analysis, of which 260 (52\%) African-American and 159 (38\%) Caucasian families had at least one diabetic member. One hundred and 57 families had at least one male diabetic member, with two families having three male diabetic members and 24 families having two diabetic members. Female diabetic members were more prevalent, as 274 families had at least one diabetic member, with one family having four diabetic members, seven families having three female diabetic members, and 42 families having two female diabetic members.

Table 1 Covariate characteristics in the combined sample and by sex for HyperGEN participants

\begin{tabular}{llll}
\hline & Complete sample & Females & Males \\
\hline Total & 3,153 & 1,883 & 1,270 \\
Diabetic, $n(\%)$ & $567(18.0)$ & $362(19.2)$ & $205(16.1)$ \\
Hypertensive, $n$ (\%) & $2,417(76.7)$ & $1,463(78.9)$ & $954(75.6)$ \\
Mean age, years (SD) & $52.6(13.6)$ & $52.3(13.6)$ & $53.1(13.7)$ \\
Race/centre, $n$ (\%) & & & \\
Caucasians, NC & $185(5.9)$ & $129(6.8)$ & $209(16.5)$ \\
Caucasians, MN & $408(12.9)$ & $199(10.6)$ & $207(16.3)$ \\
Caucasians, MA & $435(13.8)$ & $228(12.11)$ & $257(20.2)$ \\
Caucasians, UT & $517(16.4)$ & $278(13.8)$ & $118(9.3)$ \\
African-Americans, NC & $396(12.6)$ & $789(41.9)$ & $423(33.0)$ \\
African-Americans, AL & $1,212(38.4)$ & & \\
\hline
\end{tabular}

AL Alabama, MA Massachusetts, $M N$ Minnesota, NC North Carolina, UT Utah 
The multipoint adjusted genome-wide LOD scores for the combined sample and stratified by sex for all peaks with an adjusted LOD score $>1.8$ (suggestive evidence of linkage) [30] are given in Table 3. We detected a QTL influencing liability to type 2 diabetes status in females with a corresponding adjusted LOD score of 3.0 on chromosome $17 \mathrm{p} 12$ at map location $31 \mathrm{cM}$ (nearest marker AFM290VC9) (Fig. 1). This signal was considerably higher than the adjusted LOD scores observed in the complete (adjusted $\mathrm{LOD}=1.3$ ) or male (adjusted $\mathrm{LOD}=0.2$ ) samples. Three additional regions demonstrating suggestive evidence for linkage were also detected. Two were identified on chromosomes 2 and 5 in the female sample (adjusted $\mathrm{LOD}=1.8$ and 1.9 , respectively) and the third was detected on chromosome 22 (adjusted LOD $=1.9$ ) in the combined sample.

In addition, we formally assessed genotype-by-sex interaction. We found suggestive evidence of distinct additive genetic effects on type 2 diabetes status in males and females $\left(\rho_{\mathrm{g}}=0.58 \pm 0.24, p=0.051\right)$. We also assessed QTL-specific genotype-by-sex interaction at our maximum linkage peak on chromosome 17. Sizeable QTL-specific genotype-by-sex differences $(p=0.009)$ were detected on chromosome 17 at map location $31 \mathrm{cM}\left(\sigma_{q \mathrm{M}}=0.13 \pm 1.00\right.$, $\sigma_{q \mathrm{~F}}=1.23 \pm 0.92$ ).

To assess the contribution of singletons, unaffected relative pairs, and affected relative pairs (ARPs) to the linkage signal on chromosome 17, we also performed multipoint model-free ARP linkage analysis as implemented in MERLIN [31]. We used race-specific allele frequencies and examined the false-positive proportion in MERLIN using the '-simulate' command, in which 10,000 marker datasets were constructed under the null hypothesis of no linkage to type 2 diabetes. Fifty-seven AfricanAmerican and 45 Caucasian families contributed to the analysis, in which an LOD score of $2.2(p=0.0005)$ at $31 \mathrm{cM}$ was observed, thus demonstrating a substantial contribution of ARPs to the evidence for linkage. Power constraints did not facilitate ARP linkage by sex.

Table 3 Estimated adjusted LOD scores suggestive of linkage (LOD $\geq 1.8$, Rao and $\mathrm{Gu}[30]$ ) in the combined sample and by sex from multipoint quantitative trait linkage analyses of derived type 2 diabetes status in 3,153 HyperGEN participants

\begin{tabular}{lllll}
\hline Sample & Chromosome & cM & LOD & Cytogenic location \\
\hline Females & 2 & 167 & 1.8 & $2 \mathrm{q} 24.2$ \\
Females & 5 & 143 & 1.9 & $5 \mathrm{q} 31.1$ \\
Females & 17 & 31 & 3.0 & $17 \mathrm{p} 12$ \\
Combined & 22 & 28 & 1.9 & $22 \mathrm{q} 12.1$ \\
\hline
\end{tabular}

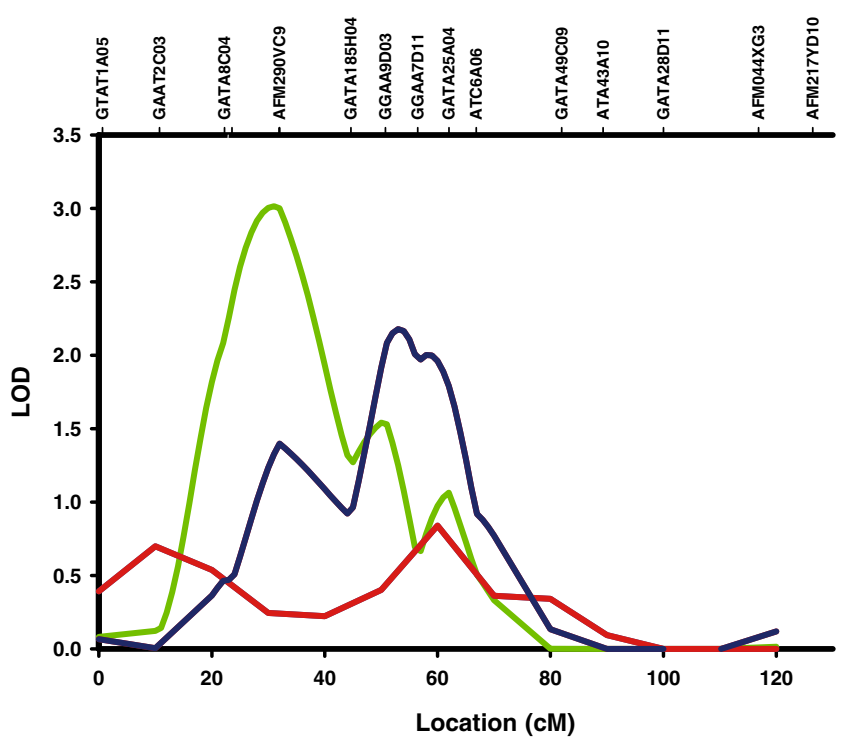

Fig. 1 Estimated robust adjusted LOD functions overall and by sex obtained from multipoint quantitative trait linkage analyses of type 2 diabetes status for chromosome 17 (green, females; red, males; blue, combined sample)

\section{Discussion}

We previously reported linkage of liability to type 2 diabetes status on chromosome 22q12.1 (adjusted $\mathrm{LOD}=1.9$ ), 8q11.23 (adjusted $\mathrm{LOD}=1.6$ ), and 17q11.2 (adjusted LOD=1.6) [10]. In this study, we extended our previous analyses to refine the localisation of these putative QTLs and further describe the genetic architecture of type 2 diabetes. Specifically, we modelled genotype-by-sex interactions, as previous work suggested strong sex effects. Indeed, modelling the true complexity of type 2 diabetes by incorporating genotype-by-sex effects may aid in the resolution of previously identified suggestive linkage peaks and improve QTL localisation by increasing the power to detect linkage [32].

We detected a QTL influencing liability to type 2 diabetes status in the female sample with a corresponding adjusted LOD score of 3.0 on chromosome 17p12. This signal was considerably higher than the adjusted LOD scores observed in the male (adjusted $\mathrm{LOD}=0.2$ ) and combined (adjusted LOD=1.3) samples.

Significant QTL-specific genotype-by-sex interaction was detected on chromosome 17 at $31 \mathrm{cM}$. Interestingly, the incorporation of sex-specific genetic effects shifted the maximum linkage peak $20 \mathrm{cM}$ towards the $\mathrm{p}$ terminus, outside the LOD drop support interval for the QTL detected in the combined sample. It is unclear which factors are influencing this shift; possibilities include low information content of the markers between the linkage peaks or two QTLs. However, an oligogenic linkage analysis did not support the presence of a second QTL on chromosome 17 
(results not shown). Our future work to identify the specific functional variants influencing liability to type 2 diabetes may consider a much larger region of chromosome 17 $(27 \mathrm{Mb})$ than previously indicated $(17 \mathrm{cM}, 6 \mathrm{Mb})$ [10], as we were unable to distinguish between poor localisation or two underlying QTLs.

To assess how robust our findings were to the partial hypertensive ascertainment correction, we also performed an ascertainment correction that accounted for the high prevalence of type 2 diabetes in this population, as families were recruited for hypertension, a trait related to type 2 diabetes. Following the approach of Cripe et al. [33], we constrained the sex-specific prevalence of type 2 diabetes using a range of population estimates (10-30\%) [34] in the polygenic model, as previous simulation analyses have demonstrated the effectiveness of this approach (J. Blangero, Southwest Foundation for Biomedical Research, San Antonio, TX, USA, personal communication, 2003). Our results indicated that the LOD scores were stable across approaches to the ascertainment correction, producing LOD scores at the same location and of similar magnitude $( \pm 1$ LOD unit, results not shown).

Differences in the genetic expression of type 2 diabetes or differences in genes contributing to the sex-related variation in disease susceptibility genes are plausible and supported by several lines of evidence. Multiple studies have suggested sexual dimorphism in type 2 diabetes rates, such as the German National Health Interview and Examination Survey [8] and the MONICA Augsburg Cohort Study [3]. Ling et al. [35] also demonstrated that insulin-stimulated peroxisome proliferator-activated receptor $\gamma$ (PPARG) coactivator $1 \alpha$ expression, a gene implicated in the activation of multiple metabolic pathways in skeletal muscle, adipose tissue, liver and pancreas, was inversely related to sex (reduced expression in females, $p<0.0001)$ in 98 non-diabetic monozygotic and dizygotic twin pairs.

Furthermore, Hegele et al. [36] demonstrated odds ratio modification by sex ( $p=0.04$ ) of $P P A R G$, a gene modulating insulin sensitivity and adipocyte differentiation, in adult Oji-Cree Canadian Indians who were homozygous for the hepatocyte nuclear factor- $1 \alpha$ G319 allele. Whereas females with at least one copy of PPARG A12 had an odds of type 2 diabetes that was 2.80 (95\% CI 1.61-4.89) times the odds of type 2 diabetes among P12 homozygotes, the estimated odds ratio for males was $0.82(95 \%$ CI $0.36-1.87)$ for the same contrast.

While we interpret the finding of significant linkages in one sex but not in the other as likely to represent sex-specific genetic effects, another and perhaps more simple explanation may be a lack of power to detect linkage in males, as $59.7 \%$ of the sample were female. However, by formally modelling a genotype-by-sex interaction, we were able to include the
2,193 pairs of relatives discordant for sex which were not included in our linkage analysis stratified by sex, thus improving our ability to detect genetic effects. Females displayed a larger QTL-specific genetic SD $(1.23 \pm 0.92)$ in comparison with males $(0.13 \pm 1.00)$, yet large SEs were noted. In addition, our simulation analyses suggested that the non-adjusted LOD scores were slightly overestimated for both males (LOD correction constant $=0.94$ ) and females (LOD correction constant $=0.91$ ).

The 1-LOD drop support interval for the signal on $17 \mathrm{p} 12$ overlaps signals from numerous genome-wide scans related to obesity measures [37-39] and one for type 2 diabetes [40]. Lindgren et al. [40] detected suggestive evidence of linkage to type 2 diabetes in 58 multiplex Finnish families on 17p12-p11 (multipoint LOD=1.9). The 17p12 locus was also implicated in several studies using variance components methods examining obesity phenotypes. Kissebah et al. [38] detected linkage to plasma leptin $(\mathrm{LOD}=5.0)$ in 2,209 individuals distributed over 507 nuclear Caucasian families, whereas Comuzzie et al. [39] identified linkage to adiponectin in 1,100 adults of predominantly northern European ancestry within 170 families (LOD=1.7). Linkage to BMI (LOD=2.47) was also observed in 753 Caucasian members of nuclear families identified through young to middle-aged probands with elevated blood pressure [37].

Several candidate genes underlie the 1-LOD unit drop support interval on chromosome $17(17 \mathrm{cM}, 6 \mathrm{Mb})$ including those for mitogen-activated protein kinase 4 (MAP2K4) and nuclear receptor corepressor 1 (NCOR1). Of note are also the ATP citrate lyase, gastrin, insulin-like growth factor-binding protein 4 , and transcription factor 2 , hepatic, genes, if we considered the larger 27-cM interval.

Lee et al. [41] demonstrated moderate differential $M A P 2 K 4$ expression in the skeletal muscle of six pairs of insulin-sensitive and insulin-resistant Pima Indians matched for percent body fat. NCOR1 has been shown to interact with all three forms of PPAR [42], well known candidate genes for type 2 diabetes. PPARG is a short-lived protein that modulates expression of several genes implicated in lipid and glucose metabolism [43]. Overexpression of NCOR1 results in a sizeable decrease of PPARG transcriptional activity. PPARG overexpression has implications in insulin resistance, as Bernal-Mizrachi et al. [44] showed that PPARG expression evoked through dexamethasone treatment in PPARG wild-type, but not PPARG-null, mice induced gluconeogenic gene expression.

No other genome-wide significant or suggestive QTLs were detected in the sample stratified by sex, although we detected suggestive evidence of linkage on chromosomes 2 and 5 in females. Further adjustment with percent body fat also recovered our previously reported QTL on 8q24.11 (adjusted $\mathrm{LOD}=2.0$ ). These suggestive linkage signals have been de- 
tected (based on varying criteria) in other genome screens of type 2 diabetes phenotypes, for example on 22q12.1 [45-47], 2q24.2 [48], 5q31.1 [49] and 8q24.11 [50].

This study may have been limited by our inability (1) to remove linear effects of additional factors influencing type 2 diabetes susceptibility, as we are investigating prevalent disease status, or (2) to stratify by other effect modifiers. We also lacked information on the age of type 2 diabetes onset for 99 participants we classified as diabetic, which may have introduced type 1 diabetic participants into our analysis. However, type 2 diabetes onset prior to age 21 was an exclusion criterion at the HyperGEN field centres during participant enrolment. We also excluded 44 individuals reporting an age at diagnosis $<30$ years, making it likely that nearly all diabetic persons were type 2 , despite missing age at onset in our subsequent analysis.

We recognise that multiple testing is a substantial issue in the analyses of gene-environment interactions. We attempted to limit the impact of this problem by focusing extensive analyses only on those regions that demonstrated differences in evidence of linkage by sex. Although this strategy led to fewer statistical tests, it may have introduced some bias. Furthermore, while we interpret the finding of significant linkages in one sex but not the other as likely to represent sex-specific genetic effects, another and perhaps more simple explanation may be that there is sufficient power to detect linkage in only one sex. Clearly further independent research is needed to confirm and refine this work.

In conclusion, we observed significant linkage results for a QTL related to the diabetic phenotype on chromosome 17 in females. We also detected suggestive evidence of linkage on chromosomes 2 and 5 in females and 22 in the complete sample. Although we detected increased support for linkage at the chromosome 17 QTL, incorporating genotype-by-sex effects did not improve QTL localisation. These signals replicate regions previously linked to type 2 diabetes status and there are plausible candidate genes for the signal detected on chromosome 17, such as MAP2K4 and NCOR1. These findings suggest that multiple genes may regulate susceptibility to type 2 diabetes and demonstrate the importance of considering the interaction of genes and environment in the aetiology of common complex traits.

Acknowledgements We acknowledge the support provided by NHLBI training grant HL007055. This work was carried out in part using computing resources at the University of Minnesota Supercomputing Institute. The hypertension network is funded by cooperative agreements (U10) with NHLBI: HL54471, HL54472, HL54473, HL54495, HL54496, HL54497, HL54509, HL54515. We thank the HyperGEN study participants. We also acknowledge the HyperGEN Participating Institutions and Principal Staff (see ESM).

We acknowledge the HyperGEN Participating Institutions and Principal Staff: Network Centre/University of Utah Field Centre:
S. Hunt, R. Williams (deceased), H. Coon, P. Hopkins, J. Hood, L. $\mathrm{Wu}$, J. Skuppin; University of Alabama at Birmingham Field Centre: A. Oberman, C. Lewis, M. Weaver, P. Johnson, S. Walker, C. Oden; Boston University/Framingham Field Centre: R. Ellison, R. Myers, Y. Zhang, L. Djoussé, J. Wilk, G. Splansky; University of Minnesota Field Centre: D. Arnett, A. Folsom, M. Miller, J. Pankow, G. Feitl, B. Lux; University of North Carolina Field Centre: G. B. Freedman, K. North, K. Rose, A. Haire; Data Coordinating Centre, Washington University: D. C. Rao, M. Province, I. Borecki, A. Adelman, D. Morgan, K. Schwander, D. Lehner, A. Kraja, S. Mandel; Central Biochemistry Lab, University of Minnesota: J. Eckfeldt, R. McGlennen, M. Tsai, C. Leiendecker-Foster, G. Rynders, J. Bucksa; Molecular Genetics Laboratory, University of Utah: M. Leppert, S. Hunt, J. Lalouel, R. Weiss; National Heart, Lung and Blood Institute: S. Old, M. Higgins (retired), C. Jaquish, M. Lundberg and M. Gerschenson.

\section{References}

1. Wilson PW, Anderson KM, Kannel WB (1986) Epidemiology of diabetes mellitus in the elderly. The Framingham Study. Am J Med 80:3-9

2. Njolstad I, Arnesen E, Lund-Larsen PG (1998) Sex differences in risk factors for clinical diabetes mellitus in a general population: a 12-year follow-up of the Finnmark Study. Am J Epidemiol 147:49-58

3. Meisinger C, Thorand B, Schneider A, Stieber J, Doring A, Lowel $\mathrm{H}$ (2002) Sex differences in risk factors for incident type 2 diabetes mellitus: the MONICA Augsburg Cohort Study. Arch Intern Med 162:82-89

4. Mitchell BD, Valdez R, Hazuda HP, Haffner SM, Monterrosa A, Stern MP (1993) Differences in the prevalence of diabetes and impaired glucose tolerance according to maternal or paternal history of diabetes. Diabetes Care 16:1262-1267

5. Murphy MJ, Metcalf BS, Voss LD et al (2004) Girls at five are intrinsically more insulin resistant than boys: the programming hypotheses revisited - the EarlyBird Study (EarlyBird 6). Pediatrics 113:82-86

6. Freedman DS, Serdula MK, Percy CA, Ballew C, White L (1997) Obesity, levels of lipids and glucose, and smoking among Navajo adolescents. J Nutr 127:2120S-2127S

7. Ehtisham S, Barrett TG, Shaw NJ (2000) Type 2 diabetes mellitus in UK children - an emerging problem. Diabet Med 17:867-871

8. Thefeld W (1999) Prevalence of diabetes mellitus in the adult German population. Gesundheitswesen 61 Spec No:S85-S89

9. Qiao Q, Hu G, Tuomilehto J et al (2003) Age- and sex-specific prevalence of diabetes and impaired glucose regulation in 11 Asian cohorts. Diabetes Care 26:1770-1780

10. Avery CL, Freedman BI, Heiss G et al (2004) Linkage analysis of diabetes status among hypertensive families: the Hypertension Genetic Epidemiology Network Study. Diabetes 53:3307-3312

11. O'Rahilly S, Barroso I, Wareham NJ (2005) Genetic factors in type 2 diabetes: the end of the beginning? Science 307:370-373

12. Williams RR, Rao DC, Ellison RC et al (2000) NHLBI family blood pressure program: methodology and recruitment in the HyperGEN network. Hypertension genetic epidemiology network. Ann Epidemiol 10:389-400

13. Pesce AJ, Kaplan LA (1987) Methods in clinical chemistry. Mosby, St Louis

14. Hinds DA, Risch N (1996) The ASPEX package: affected sib-pair exclusion mapping. Available from http://aspex.sourceforge.net/

15. Kruglyak L, Lander ES (1995) Complete multipoint sib-pair analysis of qualitative and quantitative traits. Am J Hum Genet $57: 439-454$ 
16. O'Connell JR, Weeks DE (1998) PedCheck: a program for identification of genotype incompatibilities in linkage analysis. Am J Hum Genet 63:259-266

17. Abecasis GR, Cherny SS, Cookson WO, Cardon LR (2001) GRR: graphical representation of relationship errors. Bioinformatics 17:742-743

18. Almasy L, Blangero J (1998) Multipoint quantitative-trait linkage analysis in general pedigrees. Am J Hum Genet 62:1198-1211

19. Duggirala R, Williams JT, Williams-Blangero S, Blangero J (1997) A variance component approach to dichotomous trait linkage analysis using a threshold model. Genet Epidemiol 14:987-992

20. Eisen EJ, Legates JE (1966) Genotype-sex interaction and the genetic correlation between the sexes for body weight in Mus musculus. Genetics 54:611-623

21. Comuzzie AG, Blangero J, Mahaney MC, Mitchell BD, Stern MP, MacCluer JW (1993) The quantitative genetics of sexual dimorphism in body fat measurements. Am J Hum Biol 5:725-734

22. Robertson A (1959) The sampling variance of the genetic correlation coefficient. Biometrics 15:469-485

23. Williams JT, Begleiter H, Porjesz B et al (1999) Joint multipoint linkage analysis of multivariate qualitative and quantitative traits. II. Alcoholism and event-related potentials. Am J Hum Genet 65:1148-1160

24. Williams JT, Van Eerdewegh P, Almasy L, Blangero J (1999) Joint multipoint linkage analysis of multivariate qualitative and quantitative traits. I. Likelihood formulation and simulation results. Am J Hum Genet 65:1134-1147

25. Self S, Liang K (1987) Asymptotic properties of maximum likelihood estimators and likelihood ratio tests under nonstandard conditions. J Am Stat Assoc 82:605-610

26. Comuzzie AG, Williams JT (1999) Correcting for ascertainment bias in the COGA data set. Genet Epidemiol 17 (Suppl 1):S109-S114

27. Thompson EA (1993) Sampling and ascertainment in genetic epidemiology: a tutorial review. Department of Statistics, University of Washington, Seattle, Washington

28. Blangero J, Williams JT, Almasy L (2001) Variance component methods for detecting complex trait loci. Adv Genet 42:151-181

29. Abecasis GR, Cherny SS, Cookson WO, Cardon LR (2002) Merlin - rapid analysis of dense genetic maps using sparse gene flow trees. Nat Genet 30:97-101

30. Rao DC, Gu C (2001) False positives and false negatives in genome scans. Adv Genet 42:487-498

31. Abecasis GR, Wigginton JE (2005) Handling marker-marker linkage disequilibrium: pedigree analysis with clustered markers. Am J Hum Genet 77:754-767

32. Towne B, Siervogel RM, Blangero J (1997) Effects of genotypeby-sex interaction on quantitative trait linkage analysis. Genet Epidemiol 14:1053-1058

33. Cripe L, Andelfinger G, Martin LJ, Shooner K, Benson DW (2004) Bicuspid aortic valve is heritable. J Am Coll Cardiol 44:138-143

34. Boyle JP, Honeycutt AA, Narayan KM et al (2001) Projection of diabetes burden through 2050: impact of changing demography and disease prevalence in the US. Diabetes Care 24:1936-1940

35. Ling C, Poulsen P, Carlsson E et al (2004) Multiple environmental and genetic factors influence skeletal muscle PGC-1alpha and PGC-1beta gene expression in twins. J Clin Invest 114:1518-1526
36. Hegele RA, Cao H, Harris SB, Zinman B, Hanley AJ, Anderson CM (2000) Peroxisome proliferator-activated receptor-gamma2 P12A and type 2 diabetes in Canadian Oji-Cree. J Clin Endocrinol Metab 85:2014-2019

37. Wu X, Cooper RS, Borecki I et al (2002) A combined analysis of genomewide linkage scans for body mass index from the National Heart, Lung, and Blood Institute Family Blood Pressure Program. Am J Hum Genet 70:1247-1256

38. Kissebah AH, Sonnenberg GE, Myklebust J et al (2000) Quantitative trait loci on chromosomes 3 and 17 influence phenotypes of the metabolic syndrome. Proc Natl Acad Sci U S A 97:14478-14483

39. Comuzzie AG, Funahashi T, Sonnenberg G et al (2001) The genetic basis of plasma variation in adiponectin, a global endophenotype for obesity and the metabolic syndrome. J Clin Endocrinol Metab 86:4321-4325

40. Lindgren CM, Mahtani MM, Widen E et al (2002) Genomewide search for type 2 diabetes mellitus susceptibility loci in Finnish families: the Botnia Study. Am J Hum Genet 70:509-516

41. Lee YH, Tokraks S, Pratley RE, Bogardus C, Permana PA (2003) Identification of differentially expressed genes in skeletal muscle of non-diabetic insulin-resistant and insulin-sensitive Pima Indians by differential display PCR. Diabetologia 46:1567-1575

42. Demenais F, Kanninen T, Lindgren CM et al (2003) A metaanalysis of four European genome screens (GIFT Consortium) shows evidence for a novel region on chromosome 17p11.2-q22 linked to type 2 diabetes. Hum Mol Genet 12: $1865-1873$

43. Blanquart C, Mansouri R, Fruchart JC, Staels B, Glineur C (2004) Different ways to regulate the PPARalpha stability. Biochem Biophys Res Commun 319:663-670

44. Bernal-Mizrachi C, Weng S, Feng C et al (2003) Dexamethasone induction of hypertension and diabetes is PPAR-alpha dependent in LDL receptor-null mice. Nat Med 9:1069-1075

45. Hegele RA, Sun F, Harris SB, Anderson C, Hanley AJ, Zinman B (1999) Genome-wide scanning for type 2 diabetes susceptibility in Canadian Oji-Cree, using 190 microsatellite markers. J Hum Genet 44:10-14

46. Pratley RE, Thompson DB, Prochazka M et al (1998) An autosomal genomic scan for loci linked to prediabetic phenotypes in Pima Indians. J Clin Invest 101:1757-1764

47. Sale MM, Freedman BI, Langefeld CD et al (2004) A genome-wide scan for type 2 diabetes in African-American families reveals evidence for a locus on chromosome 6q. Diabetes 53: 830-837

48. Busfield F, Duffy DL, Kesting JB et al (2002) A genomewide search for type 2 diabetes-susceptibility genes in indigenous Australians. Am J Hum Genet 70:349-357

49. Vionnet N, Hani El H, Dupont S et al (2000) Genomewide search for type 2 diabetes-susceptibility genes in French whites: evidence for a novel susceptibility locus for early-onset diabetes on chromosome 3 q27-qter and independent replication of a type 2-diabetes locus on chromosome 1q21-q24. Am J Hum Genet 67:1470-1480

50. Frayling TM, Wiltshire S, Hitman GA et al (2003) Young-onset type 2 diabetes families are the major contributors to genetic loci in the Diabetes UK Warren 2 genome scan and identify putative novel loci on chromosomes 8q21, 21q22, and 22q11. Diabetes 52:1857-1863 